\title{
Outcomes following implementation of a high-volume medical retina virtual clinic utilising a diagnostic hub during COVID-19
}

\author{
Daren Hanumunthadu ${ }^{1} \cdot$ Khadra Adan $^{1} \cdot$ Kerry Tinkler $^{1} \cdot$ Konstantinos Balaskas $^{1} \cdot$ Robin Hamilton $^{1} \cdot$ \\ Luke Nicholson ${ }^{1}$ - The Moorfields Medical Retina Virtual Assessment Study Group
}

Received: 3 January 2021 / Revised: 5 February 2021 / Accepted: 10 March 2021 / Published online: 6 April 2021

(c) The Author(s), under exclusive licence to The Royal College of Ophthalmologists 2021

\begin{abstract}
Background To describe the clinical outcomes following implementation of a high-volume medical retina virtual clinic utilising a diagnostic hub.

Methods Retrospective consecutive case-series of all patients attending the medical retina virtual clinics at Moorfields Eye Hospital (City Road) for 6 weeks from September 21, 2020.

Results In 6 weeks, 1006 patients attended the medical retina virtual clinics, which included an appointment in the diagnostic hub followed by an assessment asynchronously the following working day. The vast majority of patients were follow-up attendances $(969,96.3 \%)$ with much fewer new patient attendances $(37,3.7 \%)$. The most common diagnoses made overall were diabetic retinopathy $(457,45.4 \%)$, age-related macular degeneration $(208,20.7 \%)$ and retinal vein occlusion $(80,8.0 \%)$. The majority of patient $(643,63.9 \%)$ outcomes were follow-up in the medical retina virtual clinics including 313 (31.1\%) with OCT-only pathway and 330 (32.8\%) with OCT and widefield fundus imaging. Routine followup requested after virtual assessment included $320(31.8 \%)$ with a 3-4 month review and $267(26.5 \%)$ with a 6 months assessment. Only 62 patients $(6.2 \%)$ were asked to return for face-to-face assessment within 2 weeks.

Conclusions We describe a new high-volume medical retina virtual clinic utilising a diagnostic hub in which more than 1000 patients were seen and assessed asynchronously. Most patients were assessed as suitable for routine follow-up in this virtual pathway and only a small proportion required urgent reviews (within 2 weeks). In the COVID-19 era, this form of highvolume virtual clinic has the potential to review patients efficiently and safely.
\end{abstract}

\section{Introduction}

Virtual or remote assessments have been used successfully for several years in the United Kingdom in order to evaluate patients with glaucoma [1,2]. In addition to screening of patients for diabetic retinopathy (DR) in the United Kingdom Diabetic Eye Screening Programme (DESP), many hospitals have also developed virtual assessment clinics for

Members of the The Moorfields Medical Retina Virtual Assessment Study Group are listed below Acknowledgements.

Supplementary information The online version contains supplementary material available at https://doi.org/10.1038/s41433021-01510-4.

Luke Nicholson

luke.nicholson3@nhs.net

1 Moorfields Eye Hospital NHS Foundation Trust, London, UK the monitoring of patients with stable medical retina conditions, such as treated neovascular age-related macular degeneration (nAMD) and mild non-proliferative DR [3-5]. It is predicted that the incidence of AMD will increase by nearly 60\% between 2015 and 2035 [6]. Virtual assessment pathways have the potential to significantly help in the management of this increasing number of patients enabling hospital clinic attendances to be conserved for face-to-face assessments and treatment.

Structural assessment of macular conditions using optical coherence tomography (OCT) has become essential in the management of AMD and diabetic macular oedema (DMO) using intravitreal anti-vascular endothelial growth factor (anti-VEGF) therapy [7, 8]. Development of spectraldomain OCT (SD-OCT) has enabled faster, more detailed macular imaging improving diagnostic accuracy [9]. Significant improvements in fundus photography have emerged; the development of widefield fundus imaging (WFI) has the capacity to deliver detailed peripheral retinal 
imaging [10]. WFI using The Zeiss CLARUS Fundus camera (CLARUS 500, Carl Zeiss MediTec AG, Jena, Germany) for example provides true colour images of the retina and can deliver $200^{\circ}$ fundus coverage retinal imaging using a montage of two separate retinal images. The agreement between virtual assessment of new referrals of DR between CLARUS-delivered WFI and slit-lamp examination in a recent study of one-hundred and two eyes of 51 patients showed fair agreement between retinal grading, and importantly did not affect treatment decisions in a comparison of management outcomes [11]. The use of new retinal imaging techniques allows for detailed fundus diagnostic imaging, required in the precise evaluation of medical retina conditions and is essential to enable virtual clinic assessment, asynchronous to the time of patient attendance [12].

Previous studies have described the successful use of virtual clinic assessment in the management of the high number of stable medical retina patients [5, 13]. COVID-19 has resulted in a dramatic reduction in the number of patients seen within the hospital eye service. It is estimated that the number of medical retina patients seen during the first COVID-19 national lockdown at Moorfields Eye Hospital (MEH) was <30\% normal clinical capacity focussing on managing high-risk cases in a safe and socially distanced manner [14]. The challenge during the recovery phase was to see these large numbers of patients awaiting hospital appointments safely and efficiently, and so limit preventable visual deterioration and maintain quality of life. To this end, in the UK, The Royal College of Ophthalmologist's statement in Guidance on Restarting Medical Retina services has suggested the development of Diagnostic Hubs for patients with glaucoma and medica retina conditions [14]. By delivering high-quality imaging in dedicated Diagnostic Hubs, there is the potential to limit patient waiting times. In addition, patients have reduced appointment length and limited staff interaction at their Diagnostic Hub assessment possibly reducing exposure to transmission from the ongoing COVID-19 pandemic.

In our Diagnostic Hub model, patients attend MEH (City Road, London) for assessment that involves disease specific, trained technician-delivered history taking, visual acuity (VA), intraocular pressure (IOP) measurement and retinal imaging comprising OCT $+/$ - WFI. In nearly all cases, virtual assessment of these clinical parameters is made asynchronously with an appropriate diagnosis and management plan. The management plan is communicated to the patient by written correspondence, or telephone consultation if urgent assessment or treatment is required or further discussion is needed.

The aim of this study was to describe the clinical outcomes of the first 6 weeks of patient appointments at the medical retina digital clinics, which is a high-volume virtual medical retina clinic utilising a Diagnostic Hub approach including the rate of requirement of traditional face-to-face clinics or urgent clinical review.

\section{Materials/subjects and methods}

This was a retrospective study completed after institutional review board approval by the Audit Department of Moorfields Eye Hospital NHS Foundation Trust and adhered to the tenets set forth in the Declaration of Helsinki.

\section{Study population}

All consecutive patients attending the Medical Retina Diagnostic Hub MEH NHS Foundation Trust (City Road) for a period of 6 weeks after the commencement of asynchronous virtual assessment, between 21st September 2020 and 5th November 2020.

These medical retina patients were stratified as low or medium risk by consultant ophthalmologists in March 2020 based on their most recent clinical visit. All patients consisted of follow-up assessment except for new routine referrals from the NHS DESP for non-proliferative DR. Exclusion criteria included patients with significant cognitive impairment/ dementia, dense cataract precluding adequate fundus imaging or patients with risk of angle closure (phakic or no previous peripheral iridotomy in primary angle closure).

Medical retina conditions included DR (mild-moderate non-proliferative $\mathrm{DR}+/$ - diabetic macular edema, severe non-proliferative DR at consultant discretion, stable treated proliferative DR), stable AMD, branch retinal vein occlusion (BRVO), central serous chorioretinopathy (CSCR), macular telangiectasia and retinal artery macroaneurysm.

\section{Diagnostic hub assessment}

Figure 1 summarises the clinical pathway for patients attending the Diagnostic Hub within the medical retina service. Patients attended a technician-led assessment that includes short focussed history-taking, VA assessment and IOP measurement using rebound tonometry (iCare, Mainline instruments, UK) that is documented in the patient's electronic medical record OpenEyes (OpenEyes, London, United Kingdom). All patients were dilated with $1 \%$ tropicamide only. Patients then underwent diagnostic imaging as determined at stratification prior to patient attendance based on patient clinical diagnosis. Pathway 1 (MROCT) consisted of OCT macular volume scan and 2-field fundus imaging centred on the optic nerve and macula using the Triton or 3D/2000 (Topcon, Tokyo, Japan). Pathway 2 (MRWF) consisted of OCT macular volume scan using the Triton or 3D/2000 (Topcon, Tokyo, Japan and ultrawide 
Fig. 1 Clinical pathway for patients attending the diagnostic hub within the medical retina service. Left column: Patients attend a diagnostic hub appointment for a set of assessments and the patient returns home upon completion. Middle column: Exceptional cases are flagged and converted to a same day assessment and treatment while the rest are assessed asynchronously. Right column: Outcomes of the assessment are actioned by the administrative team.

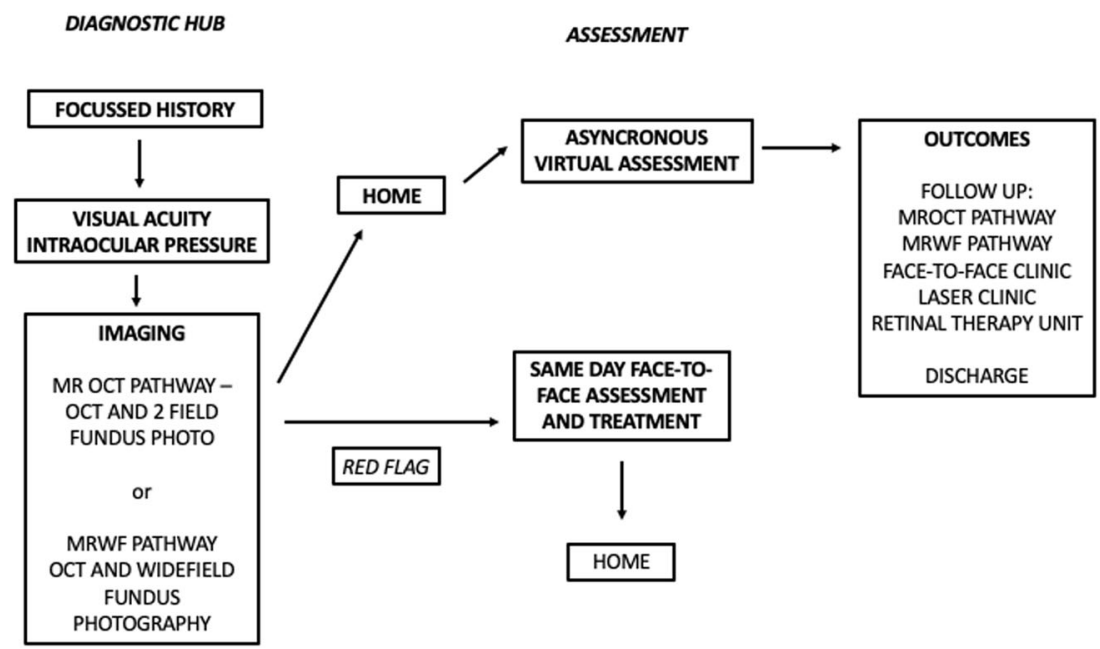

field fundus imaging (CLARUS Fundus Camera, CLARUS 500, Carl Zeiss MediTec AG, Jena, Germany).

Patients for Pathway 1 (MROCT) had diagnoses comprised of mild non-proliferative DR, stable AMD, CSCR and macular telangiectasia. Pathway 2 (MRWF) had patients with diagnoses of moderate/severe nonproliferative DR, stable treated proliferative DR and BRVO.

\section{Virtual clinic review}

All Diagnostic Hub assessments were reviewed asynchronously on the following weekday by ophthalmologists. Patients were contacted by written correspondence with a summary of their clinic evaluation; a telephone consultation was performed if the patient requires urgent clinical management. Patients were virtually assessed the same day with an option of a face-to-face consultation if they fulfilled previously denoted red flag criteria. These red flags included VA reduction of two lines on Snellen chart or more than 15 letter reduction in ETDRS (Early Treatment of Diabetic Retinopathy Study) VA, IOP $\geq 32 \mathrm{mmHg}$ and inability to obtain adequate retinal imaging.

Possible patient outcomes included further follow-up in virtual assessment in either the MROCT or MRWF pathway, or face-to-face clinic review, laser clinic, retinal therapy clinic, where intravitreal injections are administered, or discharge. Detailed protocols for our Diagnostic Hub and virtual review are available in supplementary materials.

\section{Study subject evaluation}

All patient appointments were evaluated including rates of attendance and non-attendance. Data evaluated on patient attendances included diagnosis, outcome of attendance and follow up duration. The rate of conversion to same-day face-to-face assessment and the need for telephone consultation was also assessed. All statistics were performed using Microsoft Excel (Microsoft Office for Mac, Version 11).

\section{Results}

\section{Patient attendance}

During the 6-week audit period, there were 1638 patient appointments. This included 1006 patient attendances and 632 patient non-attendances. The attendance rate for the Medical Retina Diagnostic Hub was 61.4\%. The number of patients that attended for Pathway 1 comprising OCT only (MROCT) and Pathway 2 comprising OCT and WFI (MRWF) were 517 (51.4\%) and 489 (48.6\%), respectively. The vast majority of patients were follow-up attendances $(969,96.3 \%)$ with much fewer new patient attendances $(37,3.7 \%)$.

\section{Patient diagnoses in virtual assessment}

The range of diagnoses and number $(n)$ of patients attending the medical retina virtual clinics are shown in Table 1.

The most common diagnosis made overall was DR (457, 45.4\%). DR was also the common diagnosis in the MRWF pathway $(284,58.1 \%)$ although both $\operatorname{AMD}(175,33.9 \%)$ and DR $(173,33.4 \%)$ were common in the MROCT pathway. Few patients with inherited retinal dystrophies were reviewed in the Medical Retina Digital Clinics (four patients in total).

\section{Patient outcomes}

Patient outcomes were assessed asynchronously by ophthalmologists virtually and are shown in Table 2 . 
Table 1 Diagnoses of patients attending the diagnostic hub.

\begin{tabular}{lccc}
\hline Diagnosis & $\begin{array}{l}\text { Pathway 1 (MROCT) } \\
\text { OCT only }\end{array}$ & $\begin{array}{l}\text { Pathway 2 (MRWF) OCT and } \\
\text { widefield imaging }\end{array}$ & Total \\
\hline $\begin{array}{l}\text { Age-related macular } \\
\text { degeneration }\end{array}$ & $175(17.4)$ & $33(3.3)$ & $208(20.7)$ \\
$\begin{array}{l}\text { Diabetic retinopathy } \\
\text { Retinal vein occlusion }\end{array}$ & $173(17.2)$ & $284(28.2)$ & $457(45.4)$ \\
$\begin{array}{l}\text { Central serous } \\
\text { chorioretinopathy }\end{array}$ & $31(3.1)$ & $49(4.9)$ & $80(8.0)$ \\
$\begin{array}{l}\text { Inherited retinal dystrophy } \\
\text { Other }\end{array}$ & $2(0.2)$ & $17(1.7)$ & $47(4.7)$ \\
\hline
\end{tabular}

Diagnoses of all patients ( $n, \%$ total) attending the diagnostic hub showing Pathway 1 MROCT (OCT only) and Pathway 2 MRWF (OCT and widefield imaging).
Table 2 Follow-up interval and outcomes of patients attending the diagnostic hub.

\begin{tabular}{|c|c|c|c|}
\hline & $\begin{array}{l}\text { Pathway } 1 \\
\text { (MROCT) } \\
\text { OCT only }\end{array}$ & $\begin{array}{l}\text { Pathway } 2 \\
\text { (MRWF) OCT and } \\
\text { widefield imaging } \\
(n, \%)\end{array}$ & Total \\
\hline Diagnosis & $(n, \%)$ & & $(n, \%)$ \\
\hline $\begin{array}{l}\text { Number of } \\
\text { patients }\end{array}$ & 517 & 489 & 1006 \\
\hline \multicolumn{4}{|c|}{ Follow up appointment } \\
\hline MROCT & $253(48.9)$ & $60(12.3)$ & $313(31.1)$ \\
\hline MRWF & $73(14.1)$ & $257(52.6)$ & $330(32.8)$ \\
\hline $\begin{array}{l}\text { Face to } \\
\text { face clinic }\end{array}$ & $108(20.9)$ & $116(23.7)$ & $224(22.3)$ \\
\hline Laser & $0(0.0)$ & $8(1.6)$ & $8(0.8)$ \\
\hline $\begin{array}{l}\text { Retinal } \\
\text { therapy unit }\end{array}$ & $27(5.2)$ & $17(3.5)$ & $44(4.4)$ \\
\hline Discharge & $56(10.8)$ & $31(6.3)$ & 87 (8.6) \\
\hline \multicolumn{4}{|c|}{ Follow up interval } \\
\hline $1-2$ weeks & $36(6.9)$ & $26(5.3)$ & $62(6.2)$ \\
\hline $2-4$ weeks & $25(4.8)$ & $30(6.1)$ & $55(5.5)$ \\
\hline $6-8$ weeks & $46(8.9)$ & $42(8.6)$ & $88(8.7)$ \\
\hline 3-4 months & $178(34.4)$ & $142(29.0)$ & $320(31.8)$ \\
\hline 6 months & $130(25.1)$ & $137(28.0)$ & $267(26.5)$ \\
\hline 9 months & $33(6.4)$ & $41(8.4)$ & $74(7.4)$ \\
\hline 1 year & $13(2.5)$ & $40(8.2)$ & $53(5.3)$ \\
\hline
\end{tabular}

Patients attending the diagnostic hub were assessed by ophthalmologists asynchronously and a follow up outcome of virtual assessment (Pathway 1 with OCT, Pathway 2 -OCT and widefield imaging), face-to-face clinic, laser clinic, retinal therapy unit or discharge. Follow up intervals defined were 1-2 weeks, 2-4 weeks, 6-8 weeks, 3-4 months, 6 months, 9 months and 1 year.

The majority of patients $(643,63.9 \%)$ follow-up involved continuation in the virtual assessment pathway including $313(31.1 \%)$ in the MROCT pathway and 330 $(32.8 \%)$ in the MRWF pathway. In the MROCT pathway, it appeared that the majority of patients stayed in the MROCT pathway $(253,48.8 \%)$ with much fewer patients converted to the MRWF pathway $(73,14.1 \%)$. These 73 patients included 51 with DR, 10 with RVO, 5 with AMD and 7 with other diagnoses. Of 173 patients with DR in the MROCT pathway, 59 patients $(34.1 \%)$ with were retained in the MROCT pathway, 51 (29.5\%) were reviewed in the MRWF pathway, whilst 27 with DR were converted to the face-to-face clinic for their follow up review and 18 were referred directly to the retinal therapy unit for intravitreal treatment. In the MRCWF pathway, it seemed that 257 $(52.6 \%)$ stayed in the MRWF pathway whereas just 60 (12.3\%) were converted to the MROCT pathway. This reflects previous patient stratification in that the majority of patients in the MRWF pathway were DR patients who required both WFI and OCT, whereas the AMD patients in the MROCT pathway generally require OCT imaging of the macula only for regular monitoring of disease activity. In total, $224(22.3 \%)$ patients were advised to attend for a faceto-face clinic for their next appointment; additionally, 8 $(0.80 \%)$ and $44(4.4 \%)$ of patients were followed up in the laser clinic or retinal therapy unit for direct intervention.

Telephone consultations were completed for $40(4.0 \%)$ of patients who attended the diagnostic hub assessment. Telephone consultations were also attempted for patients who did not attend or cancelled their appointments in order to help with risk stratification and decide if further appointments were necessary and in what form.

\section{Patient follow up intervals}

There were some patients in whom an urgent review was requested; this consisted of 55 (5.5\%) patients for 2-4 week review and $62(6.2 \%)$ patients for an urgent 1-2 week assessment. However, a significant number of patients had routine follow up including $320(31.8 \%)$ who required a 3-4 months review and 267 (26.5\%) who required a 6month assessment. A similar proportion required MROCT and MRWF at 3-4 months (178, 34.4\% and 142, 29.0\% respectively) and at 6 months at $(130,25.1 \%$ and 137 , $28.0 \%$ respectively). 
Otherwise, patients were assessed with outcomes that included follow-up at 6-8 weeks $(88,8.7 \%), 9$ months (74, $7.4 \%)$ and 1 year $(53,5.3 \%) .87(8.6 \%)$ patients were discharged directly from virtual assessment.

\section{Urgent assessments}

Urgent assessment was in the form of either conversion to face-to-face assessment on the day or urgent follow up in 1-2 weeks in a face-to-face clinic or direct treatment clinic (laser or RTU). Fifty-one (5.6\%) patients met at least one of the red flag criteria and were converted to direct face-to-face medical assessment on the day of their diagnostic hub assessment clinic appointment. This included 24 patients with more than 2 lines drop in vision, 9 patients with inadequate imaging, 5 patients with raised intraocular pressure and a further 13 patients in whom the cause of conversion to same-day face-to-face was not documented as due to one of the specific red flag criteria but seemed largely due to patient request for same day assessment and reassurance by a clinician. Patient diagnoses that required treatment included progression of cataract (16), reactivation of nAMD (8), DMO (6), vitreous haemorrhage (6) and cystoid macular oedema secondary to RVO (3) and ocular hypertension/ glaucoma suspect (3). Although all patients who were converted to same-day assessment were offered treatment on the day if appropriate, no patients converted to same-day assessment had a sight-threatening ocular emergency that required immediate treatment. Indeed, these patients could have been reviewed without harm within 1 week. This provides evidence to support complete asynchronous reviews without the need same day face to face reviews.

Sixty-two $(6.2 \%)$ patients were asked to return for faceto-face assessment within 2 weeks. This included 36 (58.1\%) patients attending the MROCT pathway and 26 (41.9\%) attending the MRWF pathway. This included 21 patients with AMD with suspicion of reactivation of nAMD and hence possible requirement of treatment with antiVEGF in RTU, 12 patients with DR and 2 with RVO possibly requiring further panretinal photocoagulation laser and/or anti-VEGF. The remainder of these patients comprised those in whom VA had deteriorated, assessment that further investigation including fundus fluorescein angiography was required, cataract progression was suspected or instances where patients had requested face-to-face consultation. The mean $( \pm \mathrm{SD})$ days from virtual clinic review to face to face assessment was $10.2 \pm 2.3$ days.

Fifty-five $(5.2 \%)$ patients were asked to return for assessment at 2-4 weeks. This included $25(45.5 \%)$ patients attending the MROCT pathway and $30(54.5 \%)$ patients attending the MRWF pathway. This included 18 patients in whom further treatment with intravitreal agents was required, 9 patients in whom further imaging (e.g., fundus fluorescein angiogram or OCT angiography) was required, 4 patients booked for retinal laser treatment, and 3 with poor quality imaging. The remainder consisted of patients whose vision has deteriorated subjectively, or when further assessment of new retinal imaging or listing for cataract surgery was required.

\section{Discussion}

We describe the diagnoses and outcomes of a newly developed high-volume medical retina virtual clinic service during COVID-19 by incorporating a Diagnostic Hub and next working day asynchronous virtual assessment for patients with medical retina conditions. In just under 6 weeks, more than one thousand patients attended, and clinical assessments were reviewed asynchronously the next weekday. The assessing ophthalmologists felt that it was appropriate for the vast majority of these patients to have continued follow up virtually but urgent face-to-face assessments were undertaken in those in whom it was deemed necessary. This study demonstrates the use of Diagnostic Hubs in ophthalmology in order to enable highvolume imaging with asynchronous assessment of patients in the future, particularly in the COVID-19 and postCOVID-19 era in which attempts to limit travel and attendance in hospitals for at risk patients should be encouraged along with an effective and efficient model of care to review the backlog of patients awaiting appointments.

As in other studies of virtual clinics, DR was the most common medical retina condition assessed in our study $[5,12]$. Seventy-three patients in the MROCT pathway were converted to the MRWF pathway for follow up, the majority of which comprised patients with DR $(51,69.9 \%)$ which is $29.5 \%$ of DR patients in the MROCT pathway; perhaps, all DR patients should be assessed with WFI in the future but given the high cost of widefield cameras, it is also reasonable to undertake OCT and 2-field colour fundus imaging in patients with mild non-proliferative DR. Comparison with a similar virtual assessment model for medical retina patients showed a slightly lower follow-up in virtual assessment (63.9\% in our study compared with $70.0 \%$ ) and this may reflect the recover nature of the clinics in the postCOVID era. Arguably, this is still a high level of virtual assessment follow-up; our study was completed after the first COVID-19 lockdown in which some non-urgent patients had not been seen or treated and the requirement of face-to-face assessment is possibly higher. It is anticipated that virtual assessment follow-up will increase as the recovery phase continues. Patient attendance was $61.4 \%$, lower than previous studies of virtual clinic attendance, but patient apprehension during the concurrent COVID-19 pandemic is understandable considering the age and comorbidities in the population assessed. Only 62 (6.2\%) of 
patients were asked to return for an urgent assessment within 2 weeks and were seen within $10.2 \pm 2.3$ days. It is essential for these high-volume diagnostic services to be supported by dedicated administration staff who can arrange these urgent reviews and for the hospital eye service to have the planned capacity to accept extra urgent patients that must be seen within 2 weeks.

Strengths of this study include the high volume of outcomes assessed in the recovery phase after the first nationwide lockdown after COVID-19. The majority of patients had general medical retina conditions seen regularly in ophthalmology suggesting that this model could be used elsewhere. Further study to evaluate patient-reported outcomes with this new method of assessment would be useful, as would be an assessment of patient length of appointment and number of staff interactions when they attend the Diagnostic Hub. Explanation of the new pathways is essential for patient experience; it is important to remember that some patients may benefit from a combination of face-to-face and virtual assessment over time as necessary. Significant reduction in VA was used in our virtual clinic protocol as a red flag suggesting need for urgent review. In the diagnostic hub, ETDRS letters were used to measure visual acuity using a Thompson test chart and upon entry into our electronic medical records, corresponding Snellen visual acuities are provided to the technicians. The red flag criteria included changes in VA in both Snellen and ETDRS letters as follow up patients may have had VA tested in either Snellen or ETDRS at their prior appointment. It would be sensible in the future to have a standard form of visual acuity measurement for all patients.

In the future, further development could involve more Diagnostic Hubs locally, perhaps not based at hospital sites in order that patients do not require hospital attendance and can attend locally. This is further substantiated by only $5.6 \%$ of patients having a same day face-to-face review in our model which upon review all could safely been seen urgently within a week. Virtual review of imaging including OCT and fundus photography could be completed remotely and outcomes then communicated efficiently to the patients thus reducing the footprint of medical staff within the hospital that is limited in space; if further assessment (including examination or imaging) or treatment is required, then a local follow up appointment could be generated. In the future it would be important to evaluate long-term VA outcomes of patients seen in virtual clinics compared to patients monitored in conventional face-to-face clinics.

Telephone consultations in order to discuss patient symptoms, assess treatment adherence and management plans could be used in parallel with this pathway. It is anticipated that the current COVID-19 pandemic will further stimulate the development of these 'digital' clinics in many aspects of medicine, particularly in DR [14].
Significant development in remote VA measurements has allowed the possibility of home VA assessment and could be helpful in the assessment of clinical stability, although limitation in test-retest variability in particular may hinder its general application. Home assessment of new symptoms of metamorphopsia could be used as an indicator of possible conversion of dry AMD to nAMD. The ForseeHome Device (Notal Vision, Ltd Tel Aviv Israel) for example uses preferential hyperacuity perimetry and was used to assess new metamorpopisa in a randomised controlled trial [15]. Monitoring at home using app-based technology (e.g., mVT and AllEye) are currently also being investigated as possible mechanisms to help determine symptoms of new disease activity $[16,17]$. Patients are advised to self-monitor for symptoms and to contact a dedicated email address should they experience new metamorphopsia. Due to the diagnostic hub, our clinic capacity has increased significantly, and we are able to offer reviews for patients within 1 week. In the future, with validated and robust home monitoring systems, we would anticipate that patients with detected changes via the home monitoring appliance can conveniently access the diagnostic hub for an urgent virtual assessment.

Although the need to limit patient examination, travel and hospital appointments have become more apparent with the COVID-19 pandemic, the development of teleophthalmology is likely to accelerate. The benefit of streamlined patient care in virtual assessment is clear. However, the infrastructure, funding and provision of adequately-trained staff to maintain any long-term virtual pathway is necessary. Furthermore, the technological support for electronic patient records and clinical imaging is essential; adherence to information governance is an absolute requirement. It is important that pathways for urgent review are formally arranged and patient outcomes are regularly audited.

In summary, we present the outcomes of a new teleophthalmology service for patients with medical retina conditions. In the recovery phase after the first COVID-19 lockdown, more than one thousand patients were seen and assessed; the majority of patients were then determined to continue follow up routinely in this virtual assessment pathway. Delivery of safe and efficient monitoring of medical retina conditions using diagnostic hubs in high volumes has the potential to transform the management of patients with medical retina conditions.

\section{Summary}

\section{What was known before}

- Virtual assessment has been used in ophthalmology for the assessment of glaucoma and medical retina conditions (particularly DR and age-related macular degeneration). 
- Advancements in OCT and fundus photography technology now deliver detailed imaging of the posterior segment.

\section{What this study adds}

- This retrospective study reports the clinical diagnoses and outcomes of 1006 patients attending a new highvolume medical retina virtual clinic using a diagnostic hub model and asynchronous assessment.

- Delivery of safe and efficient monitoring of medical retina conditions using diagnostic hubs in high volumes has the potential to transform the management of patients with medical retina conditions.

\section{Acknowledgements}

Funding: The authors declare no funding support or sponsorship for this study

The Moorfields Medial Retina Virtual Assessment Study Group Peter KF Addison ${ }^{1}$, Sobha Sivaprasad ${ }^{1}$, Deepthy Menon ${ }^{1}$, Barsha Sarma ${ }^{1}$, Virinder Dhillon ${ }^{1}$, Najiha Rahman ${ }^{1}$, Rajasudha Sawri-rajann, Lucia Finocchio $^{1}$, Panteleimon Fotiou ${ }^{1}$, Ricardo Romero Fontenlos ${ }^{1}$, Sandra Vermeirsch $^{1}$, Aditi Agarwal ${ }^{1}$, Hagar Khalid ${ }^{1}$, Janice Roth ${ }^{1}$, Supawat Trepatchayakom ${ }^{1}$, Maria Pilar Martin-Gutierrez ${ }^{1}$, Marcela Bohn De Albuquerque Alves ${ }^{1}$, Katarina Hurtikova ${ }^{1}$, Dana Ahnood ${ }^{1}$, Nashila Hirji $^{1}$, Rajna Rasheed ${ }^{1}$, Eleftherios Agorogiannis ${ }^{1}$, Ling Zhi Heng ${ }^{1}$, Sofia Ajamil $^{1}$, Assaf Rozenberg ${ }^{1}$, Stacey Strong ${ }^{1}$, Bahar Demir ${ }^{1}$, Carlos Valdes Lara ${ }^{1}$, Cristina Arpa ${ }^{1}$, Saqlain Sadiq ${ }^{1}$, Konstantinos Bouras ${ }^{1}$, Alexandra Hoeh ${ }^{1}$, Gabriela Grimaldi ${ }^{1}$, Karla Orsine Murta Dias ${ }^{1}$, Bejal Shah $^{1}$, Senthil Selvam ${ }^{1}$, Daren Hanumunthadu ${ }^{1}$, Elizabeth Yang ${ }^{1}$, Karen Wong $^{1}$, Charles Hennings ${ }^{1}$, Rohan Hussain ${ }^{1}$, Malgorzata Woronkowicz ${ }^{1}$

Author contributions LN and DH designed the study, collected the study data, completed the analysis and wrote the manuscript; $\mathrm{LN}$ also provided critical appraisal of the manuscript. KT, KB, RH and KA designed the study and provided critical appraisal of the manuscript. The Moorfields Medical Retina Virtual Assessment Study Group helped collect study data

\section{Compliance with ethical standards}

Conflict of interest The authors declare no competing interests.

Publisher's note Springer Nature remains neutral with regard to jurisdictional claims in published maps and institutional affiliations.

\section{References}

1. Kotecha A, Brookes J, Foster PJ. A technician-delivered 'virtual clinic' for triaging low-risk glaucoma referrals. Eye (Lond). 2017;31:899-905.
2. Ratnarajan G, Kean J, French K, Parker M, Bourne R. The false negative rate and the role for virtual review in a nationally evaluated glaucoma referral refinement scheme. Ophthalmic Physiol Opt. 2015;35:577-81.

3. Scanlon PH. The english national screening programme for diabetic retinopathy 2003-016. Acta Diabetol. 2017;54:515-25.

4. Tsaousis KT, Empeslidis T, Konidaris VE, Kapoor B, Deane J. The concept of virtual clinics in monitoring patients with age-related macular degeneration. Acta Ophthalmol. 2016;94: e353-355.

5. Kern C, Kortuem K, Hamilton R, Fasolo S, Cai Y, Balaskas K, et al. Clinical outcomes of a hospital-based teleophthalmology service: what happens to patients in a virtual clinic? Ophthalmol Retin. 2019;3:422-8.

6. Royal College of Ophthalmologists. The way forward. AgeRelated Macular Degeneration and Diabetic Retinopathy. Royal College of Ophthalmologists, London, UK; 2015.

7. Keane PA, Liakopoulos S, Jivrajka RV, Chang KT, Alasil T, Walsh AC, et al. Evaluation of optical coherence tomography retinal thickness parameters for use in clinical trials for neovascular age-related macular degeneration. Invest Ophthalmol Vis Sci. 2009;50:3378-85.

8. Zur D, Iglicki M, Busch C, Invernizzi A, Mariussi M, Loewenstein A. OCT biomarkers as functional outcome predictors in diabetic macular edema treated with dexamethasone implant. Ophthalmology. 2018;125:267-75.

9. de Boer JF, Cense B, Park BH, Pierce MC, Tearney GJ, Bouma BE. Improved signal-to-noise ratio in spectral-domain compared with time-domain optical coherence tomography. Opt Lett. 2003;28:2067-9.

10. Kiss S, Berenberg TL. Ultra widefield fundus imaging for diabetic retinopathy. Curr Diab Rep. 2014;14:514.

11. Lim WS, Grimaldi G, Nicholson L, Basheer K, Rajendram R. Widefield imaging with Clarus fundus camera vs slit lamp fundus examination in assessing patients referred from the National Health Service diabetic retinopathy screening programme. Eye (Lond). 2021;35:299-306.

12. Lee JX, Manjunath V, Talks SJ. Expanding the role of medical retina virtual clinics using multimodal ultra-widefield and optical coherence tomography imaging. Clin Ophthalmol. 2018;12:2337-45.

13. Kortuem K, Fasler K, Charnley A, Khambati H, Fasolo S, Katz M, et al. Implementation of medical retina virtual clinics in a tertiary eye care referral centre. Br J Ophthalmol. 2018;102:1391-5.

14. Royal College of Ophthalmologists. Guidance on restarting Medical Retina Services. Royal College of Ophthalmologists, London, UK; 2020.

15. Group AHSR, Chew EY, Clemons TE, Bressler SB, Elman MJ, Danis RP, et al. Randomized trial of a home monitoring system for early detection of choroidal neovascularization home monitoring of the Eye (HOME) study. Ophthalmology. 2014;121:535-44.

16. Ward E, Wickens RA, O'Connell A, Culliford LA, Rogers CA, Gidman EA, et al. Monitoring for neovascular age-related macular degeneration (AMD) reactivation at home: the MONARCH study. Eye (Lond). 2021;35:592-600.

17. Schmid MK, Thiel MA, Lienhard K, Schlingemann RO, Faes L, Bachmann LM. Reliability and diagnostic performance of a novel mobile app for hyperacuity self-monitoring in patients with age-related macular degeneration. Eye (Lond). 2019;33:1584-9. 